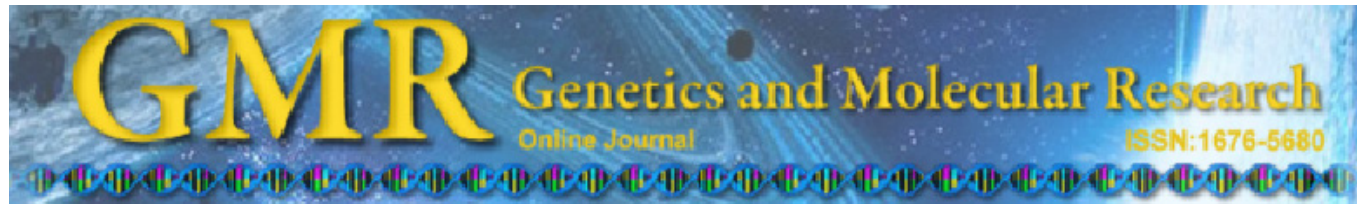

\title{
Pyrroloquinoline quinone rescues hippocampal neurons from glutamate-induced cell death through activation of Nrf2 and up-regulation of antioxidant genes
}

\author{
Q. Zhang ${ }^{1,2}$, M. Ding ${ }^{2}$, X.R. Gao ${ }^{2}$ and F. Ding ${ }^{2}$ \\ ${ }^{1}$ School of Biology and Basic Medical Sciences, Soochow University, \\ Suzhou, P.R. China \\ ${ }^{2}$ Jiangsu Key Laboratory of Neuroregeneration, University of Nantong, \\ Nantong, P.R. China
}

Corresponding author: F. Ding

E-mail: shengdd@public.nt.js.cn

Genet. Mol. Res. 11 (3): 2652-2664 (2012)

Received January 9, 2012

Accepted May 9, 2012

Published June 27, 2012

DOI http://dx.doi.org/10.4238/2012.June.27.3

\begin{abstract}
Pyrroloquinoline quinone (PQQ) has been shown to protect primary cultured hippocampal neurons from glutamateinduced cell apoptosis by scavenging reactive oxygen species (ROS) and activating phosphatidylinositol-3-kinase (PI3K)/Akt signaling. We investigated the downstream pathways of PI3K/Akt involved in PQQ protection of glutamate-injured hippocampal neurons. Western blot analysis indicated that PQQ treatment following glutamate stimulation triggers phosphorylation of glycogen synthase kinase $3 \beta$, accompanied by maintenance of Akt activation. Immunostaining and quantitative RT-PCR revealed that PQQ treatment promotes nuclear translocation of nuclear factor erythroid 2-related factor 2 (Nrf2), and up-regulates mRNA expression of Nrf2 and the antioxidant enzyme genes, heme oxygenase- 1 and glutamate cysteine ligase catalytic in glutamate-injured hippocampal neurons; this is a process dependent on the PI3K/Akt pathway, as evidenced by blocking experiments with PI3K inhibitors. In addition, increased ROS production and decreased glutathione
\end{abstract}


levels in glutamate-injured hippocampal neurons were found to be reduced by PQQ treatment. Collectively, our findings suggest that PQQ exerts neuroprotective activity, possibly through PI3K/Akt-dependent activation of $\mathrm{Nrf} 2$ and up-regulation of antioxidant genes. However, the ability of PQQ to scavenge ROS was not totally regulated by PI3K/Akt signaling; possibly it is governed by other mechanisms.

Key words: Pyrroloquinoline quinone; Reactive oxygen species; Nuclear factor erythroid 2-related factor 2; PI3K/Akt/GSK3 $\beta$ pathway; Glutamate

\section{INTRODUCTION}

Pyrroloquinoline quinone (PQQ) was initially identified as a redox enzyme cofactor, and later proven to be an essential nutrient for animal growth (Rucker et al., 2009). Recently, a body of evidence shows that PQQ plays an important role in the central and peripheral nervous system. For example, PQQ protects the brain from reversible middle cerebral artery occlusion (Zhang et al., 2006), inhibits 6-hydroxydopamine-induced neurotoxicity (Hara et al., 2007), attenuates the gene expression of inducible nitric oxide synthase in the injured spinal cord (Hirakawa et al., 2009), increases the level of nerve growth factor in astroglial cells (Murase et al., 1993), and promotes the regeneration of rat sciatic nerve (Liu et al., 2005).

Excessive production of reactive oxygen species (ROS) is considered to be involved in brain injuries and some neurodegenerative diseases. The strong ability of PQQ to scavenge ROS and attenuate oxidative stress in mitochondria may contribute to its protective effects on the nervous system (Misra et al., 2004). PQQ has been found to modulate N-methyl-D-aspartate (NMDA) receptor by directly oxidizing its redox modulatory site and to inhibit glutamateinduced ROS production in cultured cortical neurons (Aizenman et al., 1992; Scanlon et al., 1997). Our recent research demonstrated that PQQ could rescue primary hippocampal neurons from glutamate injury through inhibition of ROS production and activation of the phosphatidylinositol-3-kinase (PI3K)/Akt pathway (Zhang et al., 2011). However, the downstream pathways and the details of molecular mechanisms involved have not been fully elucidated.

One of the ways to prevent ROS-mediated cellular damage is to increase the intracellular oxidative defense capacity. There is a wide variety of factors associated with the cellular response to oxidative stress. Nuclear factor erythroid 2-related factor $2(\mathrm{Nrf} 2)$ is important for the cell to fight oxidative stress, and so neural cells from $\mathrm{Nrf2}$ knockout $\left(\mathrm{Nrf2}^{--}\right)$mice are more vulnerable to oxidative stress than normal (Dhakshinamoorthy et al., 2000; Lee and Johnson, 2004; Niture et al., 2010). Nrf2 binds to the antioxidant response element (ARE) and regulates the expression of ARE-mediated antioxidant enzyme genes, such as heme oxygenase-1 (HO1) and glutamate cysteine ligase catalytic (GCLC), in response to a variety of oxidative stimuli (Ishii et al., 2000; Kaspar et al., 2009).

In this study, we aimed to investigate the downstream pathways of PI3K/Akt involved in PQQ protection of glutamate-injured hippocampal neurons to further elucidate the molecular mechanisms underlying the neuroprotective effects of PQQ. We noticed that PQQ treatment triggered the phosphorylation of glycogen synthase kinase $3 \beta$ (GSK3 $\beta$ ) accompanied by the maintenance of Akt activation. To gain insight into the functional interactions between 
the PI3K/Akt/GSK3 $\beta$ pathway and Nrf2-dependent antioxidant system, we examined the possible influences of PQQ treatment on activation of Nrf2 and subsequent expression changes of antioxidant genes HO-1 and GCLC in glutamate-injured hippocampal neurons, and identified the relationship between these influences and the PI3K/Akt/GSK3 $\beta$ pathway. In addition, we observed that the ability of PQQ to scavenge ROS, generated for protection of hippocampal neurons against glutamate injury, was not totally regulated by the PI3K/Akt signaling.

\section{MATERIAL AND METHODS}

\section{Materials}

PQQ, trypsin, wortmannin (an inhibitor of PI3K), poly-L-lysine, Hoechst 33342, 2',7'-dichlorofluorescin diacetate (DCFH-DA), monoclonal rabbit anti-phospho-Akt (Ser473) antibody, monoclonal mouse anti- $\beta$-actin antibody, monoclonal mouse anti-MAP2 antibody, and L-glutamate were purchased from Sigma (St. Louis, MO, USA). LY294002 (an inhibitor of PI3K), monoclonal mouse anti-protein kinase $\mathrm{B} \alpha(\mathrm{PKB} \alpha / \mathrm{Akt})$ antibody, polyclonal rabbit anti-phospho-GSK3 $\beta$ antibody, monoclonal mouse anti-GSK3 $\beta$ antibody, and polyclonal rabbit anti-Nrf2 antibody were purchased from Cell Signaling (Danvers, MA, USA). Neurobasal medium, Dulbecco's modified Eagle's medium (DMEM), B27 supplement, and fetal bovine serum (FBS) were purchased from Gibco (Grand Island, NY, USA). IRDye 800-conjugated goat anti-mouse IgG and IRDye 800-conjugated donkey anti-rabbit IgG were purchased from Rockland (Gilbertsville, PA, USA). Donkey anti-rabbit IgG-FITC and goat anti-mouse IgG-TRITC were purchased from Santa Cruz (Santa Cruz, CA, USA). Cell lysis buffer, protease inhibitor cocktail and the BCA-based protein quantification kit were obtained from Biocolor (Shanghai, China). The Trizol reagent was obtained from Invitrogen (Carlsbad, CA, USA). The omniscript Reverse Transcription (RT) kit was from Qiagen (Valencia, CA, USA). Fast EvaGreen qPCR Master Mix was from Biotium (Hayward, CA, USA). The assay kit for glutathione (GSH) was obtained from Jiancheng Institute of Biotechnology (Nanjing, Jiangsu, China).

\section{Cell culture and cell treatment}

Hippocampal neurons were harvested from E18.5 Sprague-Dawley (SD) rat embryos (obtained from the Experimental Animal Center of Nantong University, China) exactly as described previously (Zhang et al., 2011). Briefly, the rat brain was dissected to collect the hippocampi, which were subjected to digestion with $0.25 \%$ trypsin in $\mathrm{Ca}^{2+}$ - and $\mathrm{Mg}^{2+}$-free Hank's balanced salt solution at $37^{\circ} \mathrm{C}$ for $10 \mathrm{~min}$. The cells re-suspended in DMEM supplemented with $10 \%$ FBS were plated onto a poly-L-lysine-coated plate at a density of $1 \times 10^{5}$ cells $/ \mathrm{cm}^{2}$ and the medium was replaced by Neurobasal medium supplemented with 2\% B27, $0.5 \mathrm{mM}$ glutamine, $100 \mathrm{U} / \mathrm{mL}$ penicillin, and $100 \mu \mathrm{g} / \mathrm{mL}$ streptomycin after cell attachment. After incubation in a humidified atmosphere of $95 \%$ air and $5 \% \mathrm{CO}_{2}$ at $37^{\circ} \mathrm{C}$ for $7 \sim 8$ days, primary cultured hippocampal neurons were treated with $125 \mu \mathrm{M}$ glutamate for 15 min to establish a cell injury model. The cells were washed with phosphate-buffered saline (PBS, $0.01 \mathrm{M}, \mathrm{pH} 7.4)$, followed by different concentrations of PQQ treatment for different times as indicated. Two PI3K/Akt inhibitors, LY294002 $(10 \mu \mathrm{M})$ and wortmannin $(100 \mathrm{nM})$ were added to the culture with PQQ, respectively, to observe whether the activation of PI3K/Akt 
was involved in the effects of PQQ. The hippocampal neurons receiving only vehicle treatment served as control. After specified cell treatments, different measurements were performed as described below.

\section{Western blot analysis}

Cultured hippocampal neurons were collected for homogenization in a cell lysis buffer consisting of $62.5 \mathrm{mM}$ Tris, $\mathrm{pH}$ 6.8, $10 \mathrm{mM}$ dithiothreitol (DTT), 2\% sodium dodecyl sulfate (SDS), $10 \%$ glycerol and a dilution of a protease inhibitor cocktail (100 mM phenylmethanesulfonyl fluoride, $15 \mu \mathrm{M}$ aprotinin, $100 \mu \mathrm{M}$ leupeptin, $100 \mu \mathrm{M}$ bestatin, $100 \mu \mathrm{M}$ pepstatin, and $80 \mu \mathrm{M}$ E-64 diluted in dimethyl sulfoxide). Total protein was quantified by BCA analysis and separated by electrophoresis on SDS-PAGE gels. After transferring to a PVDF membrane (Millipore, Bedford, MA, USA), the membrane was blocked with 5\% non-fat dry milk in Tris-buffered saline (TBS, $\mathrm{pH}$ 7.4) and incubated with anti-phospho-Akt (p-Akt, 1:500), anti-total-Akt (t-Akt, 1:1000), anti-phospho-GSK3 $\beta$ (p-GSK3 $\beta, 1: 1000$ ) or anti-total-GSK3 $\beta$ (t-GSK3 $\beta, 1: 1000$ ), at $4^{\circ} \mathrm{C}$ overnight. After washing with TBS/T (TBS with $0.1 \%$ Tween 20 ), IRDye 800-conjugated affinity purified goat anti-mouse $\operatorname{IgG}(1: 5000)$ or donkey anti-rabbit $\operatorname{IgG~(1:5000)~was~applied~at~room~temperature~for~} 30 \mathrm{~min}$. The images were scanned with Odyssey infrared imaging system (LI-COR Biosciences, Lincoln, NE, USA), and the data were analyzed with the PDQuest 7.2.0 software (Bio-Rad, Berkeley, CA, USA). $\beta$-actin $(1: 4000)$ was used as an internal control protein for equal loading.

\section{Immunofluorescence staining}

Cultured hippocampal neurons were fixed with $4 \%$ paraformaldehyde for $30 \mathrm{~min}$. After 3 washes in PBS, the cells were permeabilized with 5\% Triton X-100 and incubated in $0.3 \%$ goat serum (in PBS) for $30 \mathrm{~min}$ at $37^{\circ} \mathrm{C}$. Primary antibodies against $\mathrm{Nrf2}$ (1:200) and MAP2 (1:200) were applied overnight at $4{ }^{\circ} \mathrm{C}$, followed by staining with donkey antirabbit IgG-FITC (excitation at $488 \mathrm{~nm}$ and emission at $520 \mathrm{~nm}, 1: 200$ ) and goat anti-mouse IgG-TRITC (excitation at $544 \mathrm{~nm}$ and emission at $572 \mathrm{~nm}, 1: 200$ ) at room temperature for $2 \mathrm{~h}$. The nuclei were stained with Hoechst 33342 (excitation at $340 \mathrm{~nm}$ and emission at 510 $\mathrm{nm}$ ). The images were acquired by confocal laser scanning microscopy and photographed (Leica, Heidelberg, Germany).

\section{Quantitative reverse transcription-PCR (qPCR)}

Total RNA was extracted from cultured hippocampal neurons with the Trizol reagent following manufacturer instructions. Briefly, $100 \mathrm{ng}$ total RNA was reverse transcribed into cDNA with the Omniscript RT kit according to manufacturer instructions, and qPCR was carried out using the Fast EvaGreen ${ }^{\circledR}$ qPCR Master Mix. The reaction mixtures contained $10 \mu \mathrm{L} 2 \mathrm{X}$ Fast EvaGreen Master Mix, $2 \mu \mathrm{L}$ ROX, $0.25 \mu \mathrm{M}$ each primer (as listed in Table 1), and $1 \mu \mathrm{L}$ cDNA. The PCR amplifications were performed with the 7300 Realtime PCR system (Applied Biosystems, Foster City, CA, USA). The relative expression of each mRNA was calculated using the comparative $2^{-\Delta \Delta C t}$ method, and normalized by that of $\beta$-actin. 
Table 1. Oligonucleotide sequences used in quantitative reverse transcription-PCR analysis.

\begin{tabular}{llc}
\hline Gene & Primer sequence $\left(5^{\prime}-3^{\prime}\right)$ & Amplicon size (bp) \\
\hline Nrf1 & Forward - CCCTCAAGAAAGGTAGCAV & 169 \\
Nrf2 & Reverse - GCCTCGCTCAACTGGTAT & 190 \\
& Forward - ACATTCAAGCCGATTAGAGG & 111 \\
HO-1 & Reverse - GAAGTCATCAACAGGGAG & 11 \\
GCLC & Forward - TTTCACCTTCCCGAGCAT & 159 \\
& Reverse - GCCTCTTCTGTCACCCTGT & 159 \\
& Forward - GAGTAGAGTTCCGACCAAT & 118 \\
& Reverse - GCTCCTGTGCCACTTTCA & 118 \\
\hline
\end{tabular}

\section{ROS assay}

The intracellular ROS level was determined by the DCFH-DA assay. The cells were incubated with DCFH-DA at a final concentration of $20 \mu \mathrm{M}$ at $37^{\circ} \mathrm{C}$ for $30 \mathrm{~min}$ in the dark, and then gently rinsed with PBS 3 times. ROS levels were measured as the fluorescence of oxidation product of DCFH-DA, dichlorofluorescin (DCF), with excitation and emission wavelengths of 485 and $528 \mathrm{~nm}$ detected by a multi-mode microplate reader (Bio-Tek, Winooski, VT, USA).

\section{GSH measurement}

Cultured hippocampal neurons were collected for homogenization in a cell lysis buffer, followed by GSH measurement with a special kit according to manufacturer instructions. GSH reacted with 5, 5'-dithiobis-2-nitrobenzoic acid (DTNB) to generate 2-nitro-5-thiobenzoic acid, which is a yellow-colored product with maximum absorbance at $405 \mathrm{~nm}$. An equal volume of double-distilled $\mathrm{H}_{2} \mathrm{O}$ was used as a blank. The GSH content was calculated from a standard curve.

\section{Statistical analysis}

All data were presented as means \pm SEM for three independent experiments. Comparisons were analyzed by one-way analysis of variance (ANOVA) and subsequently the Tukey multiple comparison test with the Stata 6.0 package software (Stata Corp., College Station, TX, USA). $\mathrm{P}<0.05$ was considered statistically significant.

\section{RESULTS}

\section{PQQ activated the phosphorylation of Akt/GSK3ß pathway}

Our previous study showed that treatment with 50 or $100 \mu \mathrm{M}$ PQQ for 30 min could significantly increase the phosphorylated Akt level in glutamate-injured hippocampal neurons (Zhang et al., 2011). In this study, we treated glutamate-injured hippocampal neurons with 100 $\mu \mathrm{M}$ PQQ for different times (5 and $30 \mathrm{~min}$, and 2, 4, and $24 \mathrm{~h}$ ), and investigated whether the activation of Akt was transient or sustained. As indicated by Western blot analysis (Figure 1A and B), if no PQQ was applied, the phosphorylation of Akt (phospho-Akt/total-Akt) increased at 5 min after glutamate injury, and then gradually decreased with time to a normal level; if PQQ was applied after glutamate injury, the phosphorylation of Akt peaked at $30 \mathrm{~min}$, and then gradually decreased with time. The phospho-Akt/total-Akt ratio in glutamate-injured hip- 
pocampal neurons was significantly higher at $30 \mathrm{~min}$ or $2 \mathrm{~h}$ of PQQ treatment than at $30 \mathrm{~min}$ or $2 \mathrm{~h}$ of no treatment, respectively.

Since GSK3 $\beta$ is one of the major downstream targets of Akt pathway, we tested whether the phosphorylation of GSK3 $\beta$ was also regulated by PQQ treatment. Also, as indicated by Western blot analysis (Figure 1A and C), if no PQQ was applied, the phosphorylation of GSK3 $\beta$ (phospho-GSK3 $\beta /$ total-GSK3 $\beta$ ) showed no significant time-dependent changes over the time period studied after glutamate injury; if PQQ was applied after glutamate injury, the phosphorylation of GSK $3 \beta$ peaked at $30 \mathrm{~min}$ and then gradually decreased with time to a normal level. The phospho-GSK3 $\beta /$ total-GSK3 $\beta$ ratio in glutamate-injured hippocampal neurons was significantly higher at $30 \mathrm{~min}$ of PQQ treatment than at $30 \mathrm{~min}$ of no treatment. These data indicated that Akt/GSK3 $\beta$ signaling was affected by PQQ in glutamate-injured neurons and the activation of Akt was sustained.
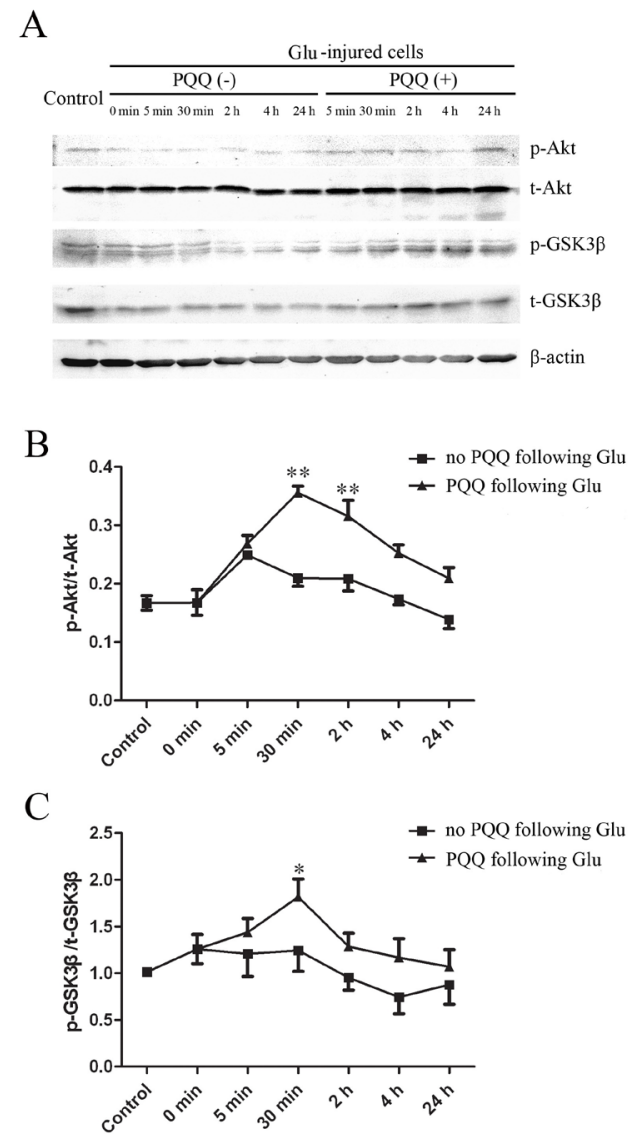

Figure 1. Phyrroloquinoline quinone (PQQ) affected phosphorylation of Akt and GSK3 $\beta$ in glutamate (Glu)injured hippocampal neurons. The ratios of phospho-Akt (p-Akt) to total-Akt (t-Akt) (B) and phospho-GSK3 $\beta$ $(\mathrm{p}-\mathrm{GSK} 3 \beta)$ to total-GSK3 $\beta$ (t-GSK3 $\beta$ ) $(\mathbf{C})$ were detected when Glu $(125 \mu \mathrm{M})$-stimulated (for $15 \mathrm{~min}$ ) hippocampal neurons received or did not receive PQQ $(100 \mu \mathrm{M})$ treatment for different times $(0,5$, and $30 \mathrm{~min}, 2,4$, and $24 \mathrm{~h})$, respectively. Primary cultured hippocampal neurons receiving only vehicle treatment served as control. $* \mathrm{P}<0.05$ and ${ }^{*} * \mathrm{P}<0.01$ versus receiving no PQQ treatment at the same time. $\mathbf{A}$ is the representative Western blot images, in which $\beta$-actin served as an internal control. 


\section{PQQ promoted the translocation of Nrf2 to nucleus dependent on activation of PI3K/Akt pathway}

Double immunostaining with anti-MAP2 (a neuronal marker) and anti-Nrf2 displayed the spatial pattern of $\mathrm{Nrf} 2$ expression in hippocampal neurons after different cell treatments. There was little expression of Nrf2 in the nucleus of control cells (Figure 2A). PQQ treatment alone induced the nuclear translocation of $\mathrm{Nrf2}$ to a certain extent (Figure 2B). Glutamate stimulation alone hardly affected the expression pattern of $\mathrm{Nrf} 2$ despite a great damage to neurites as shown by the loss of MAP2 staining (Figure 2C). PQQ treatment following glutamate stimulation rescued neurite damage and promoted the translocation of $\mathrm{Nrf} 2$ from the cytoplasm to nucleus (Figure 2D). We previously demonstrated that PI3K/Akt inhibition by LY294002 or wortmannin significantly blocked the protective effects of PQQ (Zhang et al., 2011). In this study, we found that these two PI3K inhibitors also blocked the influence of PQQ on Nrf2 translocation, implying that PI3K/Akt activation was required for the nuclear translocation of Nrf2 in glutamate-injured hippocampal neurons (Figure 2E and F).
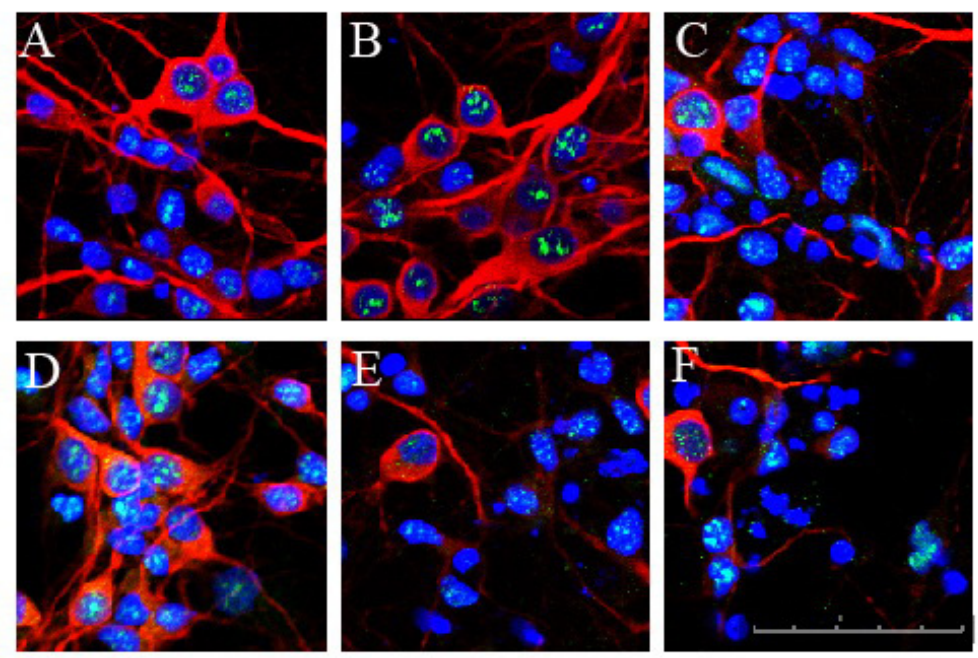

Figure 2. Phyrroloquinoline quinone (PQQ) treatment promoted the Nrf2 translocation to the nucleus in glutamateinjured hippocampal neurons and phosphatidylinositol-3-kinase (PI3K/Akt) pathway was involved. Primary cultured hippocampal neurons were stimulated with $125 \mu \mathrm{M}$ Glu for 15 min and then treated with $100 \mu \mathrm{M}$ PQQ with or without PI3K inhibitor for $24 \mathrm{~h}$, followed by immunofluorescence staining of MAP2 (red) and Nrf2 (green). The nucleus was stained with Hoechst 33342 (blue). A. Control; B. PQQ $(100 \mu \mathrm{M})$; C. Glu $(125 \mu \mathrm{M})$; D. Glu (125 $\mu \mathrm{M})+$ PQQ $(100 \mu \mathrm{M})$; E. Glu $(125 \mu \mathrm{M})+$ PQQ $(100 \mu \mathrm{M})+$ LY294002 $(10 \mu \mathrm{M}) ; \mathbf{F}$. Glu $(125 \mu \mathrm{M})+$ PQQ $(100 \mu \mathrm{M})$ + wortmannin $(100 \mathrm{nM}) . \mathrm{Bar}=50 \mu \mathrm{M}$.

\section{PQQ altered the mRNA expressions of Nrf2, HO-1 and GCLC}

The qPCR data showed the changes in mRNA expressions of Nrf1, Nrf2, HO-1, and GCLC. Different cell treatments did not significantly alter the mRNA expression of Nrf1 in cultured hippocampal neurons (Figure 3A). In contrast, the mRNA expression of Nrf2 in cultured hippocampal neurons was significantly decreased by glutamate stimulation, and then was significantly increased by PQQ treatment in a dose-dependent manner (Figure 3B). Either 
of the PI3K inhibitors, LY294002 or wortmannin, attenuated the PQQ-induced up-regulation of Nrf2 mRNA in glutamate-injured hippocampal neurons (Figure 3B). Likewise, the mRNA expression of HO-1 and GCLC, two target genes of Nrf2, in glutamate-injured hippocampal neurons were also up-regulated by PQQ treatment, and then attenuated by concomitant use of PI3K inhibitor, LY294002 or wortmannin. These qPCR data suggested that the up-regulation of Nrf2 and ARE-mediated antioxidant enzyme genes may be responsible for the neuroprotective action of PQQ and that such up-regulation is also dependent on the activation of PI3K/ Akt signal pathway.

A

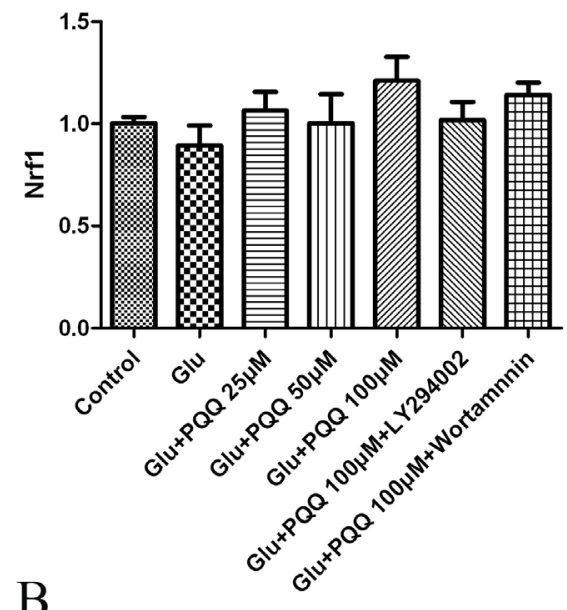

$\mathrm{B}$

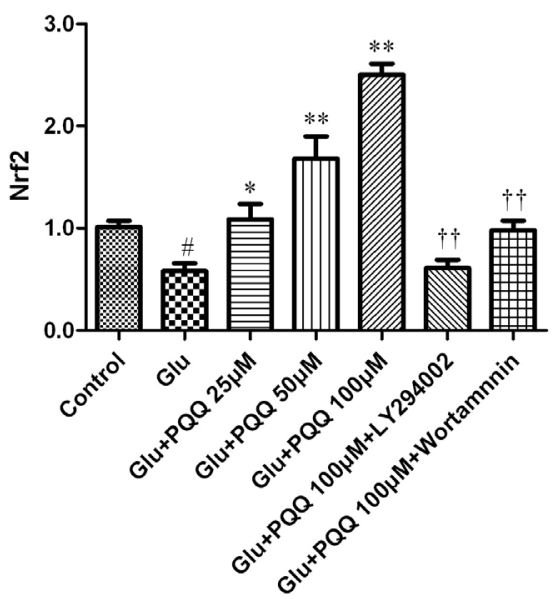

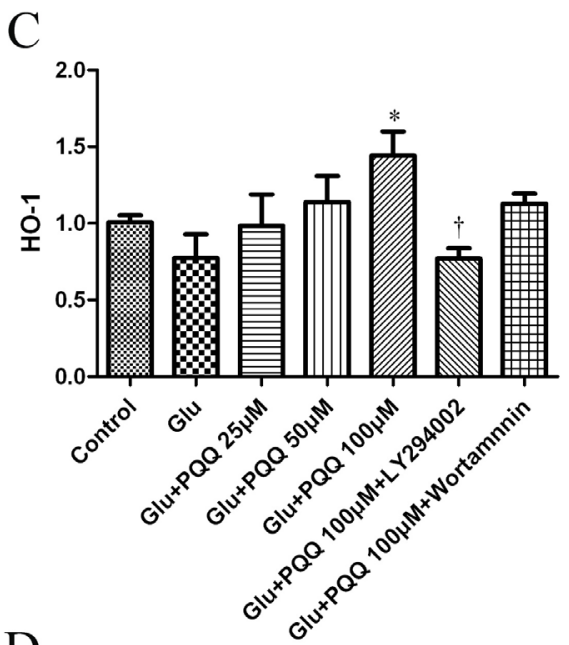

D

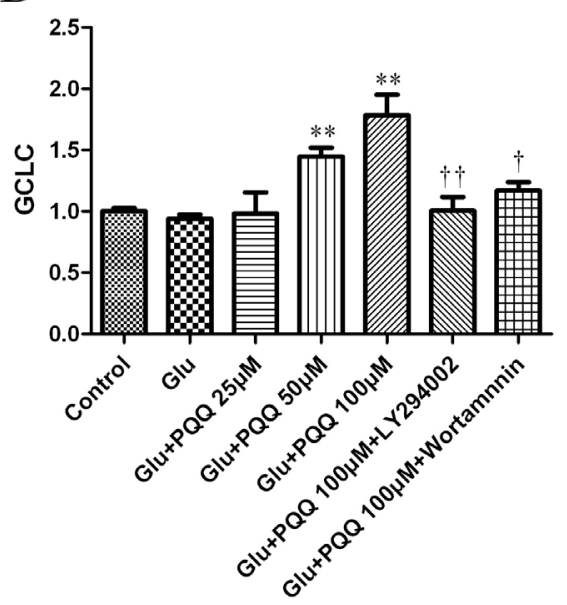

Figure 3. mRNA expressions of transcription factors and enzymes affected by phyrroloquinoline quinone (PQQ) treatment dependent of PI3K/Akt pathway. The qPCR data of the mRNA of Nrf1 (A), Nrf2 (B), HO-1 (C), and GCLC (D) in cultured hippocampal neurons stimulated by $125 \mu \mathrm{M}$ glutamate (Glu) for $15 \mathrm{~min}$ and then treated with PQQ $(25,50$ and $100 \mu \mathrm{M})$ for $30 \mathrm{~min}$ with or without PI3K inhibitors LY294002 $(10 \mu \mathrm{M})$ and wortmannin $(100$ $\mathrm{nM})$, respectively. ${ }^{*} \mathrm{P}<0.05$ and ${ }^{*} * \mathrm{P}<0.01$ versus Glu $(125 \mu \mathrm{M})$ stimulation alone, ${ }^{\sharp} \mathrm{P}<0.01$ versus control, ${ }^{\dagger} \mathrm{P}<$ 0.05 and ${ }^{\dagger}<0.01$ versus Glu $(125 \mu \mathrm{M})+$ PQQ $(100 \mu \mathrm{M})$. 


\section{PQQ inhibited ROS production and increased GSH level}

Intracellular accumulation of ROS has been implicated in the pathogenesis of several disease states. In this study, the ROS level was measured by a cell-permeable fluorogenic probe DCFH-DA, which could diffuse into cells for deacetylation by cellular esterases into non-fluorescent DCFH, which would then be rapidly oxidized by ROS into highly fluorescent DCF. Fluorescence intensity in this assay was proportional to the ROS level within the cell cytosol. Glutamate-injured hippocampal neurons displayed a large amount production of ROS, as verified by an increase in the fluorescence intensity of DCF. Although PQQ treatment alone did not affect ROS production, PQQ treatment following glutamate injury could significantly reduce ROS production to some extent (Figure 4A).

GSH is one of the most important molecules in mammalian cells for scavenging ROS. In this study, the GSH level was measured by the DTNB method. After glutamate injury, the GSH level was reduced to about $70 \%$ of normal, and then recovered approaching the normal level by the subsequent PQQ treatment (Figure 4B).

Inhibition of PI3K by either PI3K inhibitor failed to alter the effect of PQQ on ROS production or GSH level (Figure 4A and B), suggesting that the activation of PI3K/Akt signaling was not required in the PQQ-induced regulation of ROS and GSH; in other words, some of other signaling molecules might have been involved.

A

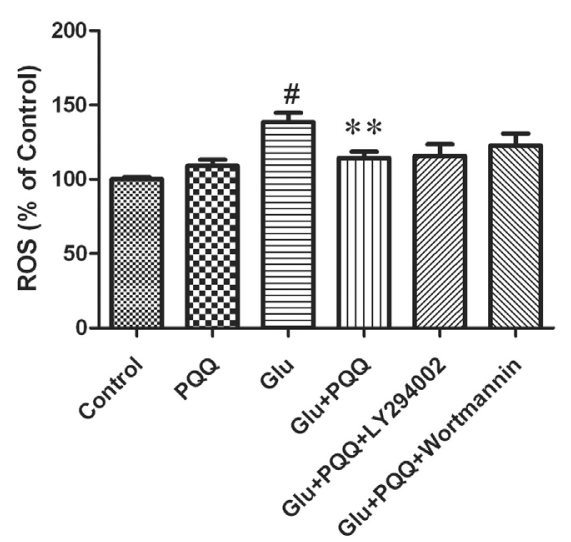

B

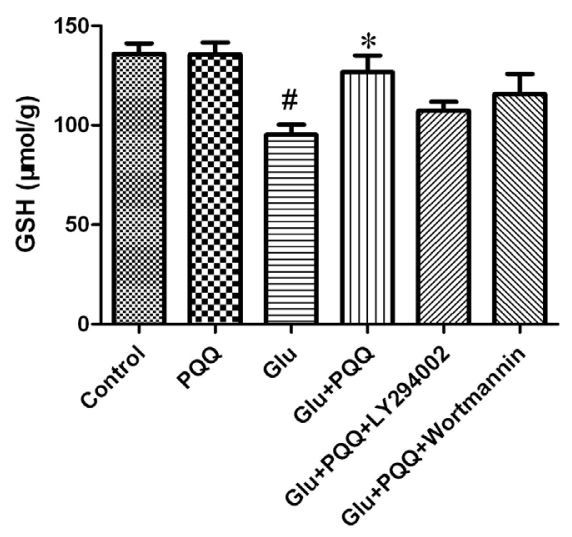

Figure 4. Reactive oxygen species (ROS) production and GSH level affected by phyrroloquinoline quinone (PQQ) treatment independent of PI3K/Akt pathway. Primary cultured hippocampal neurons were stimulated by $125 \mu \mathrm{M}$ glutamate (Glu) for $15 \mathrm{~min}$, and then treated with $100 \mu \mathrm{M}$ PQQ with or without PI3K inhibitors LY294002 (10 $\mu \mathrm{M})$ and wortmannin $(100 \mathrm{nM})$. After 30-min incubation, ROS production was measured by DCFH-DA assay (A), and after 24-h incubation, GSH level was determined by DTNB method (B). ${ }^{*} \mathrm{P}<0.05$ and ${ }^{* *} \mathrm{P}<0.01$ versus Glu (125 $\mu \mathrm{M})$ stimulation alone. ${ }^{\#} \mathrm{P}<0.01$ versus control.

\section{DISCUSSION}

PQQ treatment can rescue primary cultured hippocampal neurons from glutamate injury (Zhang et al., 2011). For further elucidation of mechanisms involved, this study showed that PQQ treatment following glutamate stimulation led to the sustained activation of Akt and 
downstream phosphorylation of GSK3 $\beta$, promoted the PI3K/Akt pathway-dependent nuclear translocation of transcription factor $\mathrm{Nrf} 2$, and resulted in the up-regulation of mRNA expression of transcription factor Nrf2 and antioxidant enzyme genes, HO-1 and GCLC, which was also dependent on the activation of the PI3K/Akt pathway. In addition, we found that the increase in ROS production and decrease in GSH level in glutamate-injured hippocampal neurons were both antagonized by PQQ treatment.

PQQ has been reported to display a strong anti-apoptotic activity in cortical neurons and to activate multiple cell survival pathways that are essential to cell growth, survival and differentiation, such as Ras/ERK and PI3K/Akt signaling (Kumazawa et al., 2007; Mebratu and Tesfaigzi, 2009; Zhang et al., 2011). As is known, the down-regulation of GSK3 $\beta$ activity is one of the mechanisms involved in cell survival, promoting the function of PI3K/Akt signaling pathway (Pap and Cooper, 1998). Contrary to most signaling kinases, GSK3 $\beta$ is active in unstimulated cells and makes cells sensitive to insults. The activation of signaling kinases such as Akt, extracellular regulated protein kinases (ERK), and protein kinase C (PKC), may phosphorylate GSK3 $\beta$ at Ser9, leading to its inactivation (Cross et al., 1995). In this study, we found that glutamate stimulation induced a short-term ( $5 \mathrm{~min}$ ) activation of Akt, implying that some internal processes of protecting cells from death may be switched on. However, this activation of Akt by glutamate was instant and the activated signal was not enough for the cells to survive the injury. In contrast, PQQ treatment could lead to a sustained (more than $2 \mathrm{~h}$ ) phosphorylation of Akt, which may provide essential conditions to antagonize glutamate-induced cell apoptosis. Meanwhile, GSK3 $\beta$ was phosphorylated by PQQ with a peak at 30 min, suggesting that GSK3 $\beta$ may be one of the downstream signaling molecules of Akt in our experimental setting.

GSK3 $\beta$ plays an important role in the nuclear exclusion and inactivation of the transcription factor Nrf2, resulting in the sensitization of neurons to oxidative damage. Functional interference between GSK3 $\beta$ and Nrf2 protects hippocampal neurons against kainate-induced cell death (Salazar et al., 2006; Rojo et al., 2008). Under basal conditions, Nrf2 binds tightly to the regulatory protein Kelch-like ECH-associated protein 1 (Keap1) and retains it in the cytoplasm. The phosphorylation of Nrf2 at Ser40 by PKC causes the dissociation of Nrf2 from Keap1 and subsequent translocation to the nucleus (Huang et al., 2002). After translocation, Nrf2 activates a battery of ARE-driven genes in the nucleus, including direct antioxidant enzymes (such as catalase, superoxide dismutase and GSH) and indirect antioxidant enzymes (such as phase II detoxifying enzymes, GSH generation enzymes and stress response proteins), and several specific chemicals exhibit antioxidant, ROS-eliminating properties through Nrf2 signaling (Jung and Kwak, 2010). In this study, we observed that PQQ treatment promoted the translocation of $\mathrm{Nrf} 2$ from the cytoplasm to nucleus in glutamate-injured hippocampal neurons, and that the application of PI3K inhibitors blocked this nuclear translocation. These data suggested that Nrf2 translocation might have played an important role in the cell survival promoted by PQQ and that PI3K/Akt/GSK3 $\beta$ was required for this process.

It has been reported that PQQ can stimulate the activation of cAMP response elementbinding protein (CREB) in combination with some transcription factors such as Nrf1, Nrf2 and mitochondrial transcription factors, enhancing mitochondriogenesis (Bauerly et al., 2006; Stites et al., 2006; Zhu et al., 2006; Chowanadisai et al., 2010), whereas the Nrf2/ARE signaling pathway has been recognized as a key contributor to the cellular response to neuronal injury in vitro and in vivo (Shih et al., 2005). In this study, we measured the expression level of Nrf2 and some related transcript factors to demonstrate whether Nrf2/ARE signaling was influenced by PQQ treatment. 
Nrf1 and Nrf2 are members of the Cap'n'Collar (CNC) family of transcription factors and both of them bind to ARE (Motohashi et al., 2002; Blank, 2008). Because of the similarity in binding specificity and expression profile between Nrf1 and Nrf2, they are supposed to share overlapping target genes and overlapping functions. However, Nrf1 or Nrf2 knock-out mice display distinct phenotypes because of the expression differences in their relevant target genes (Itoh et al., 1997; Kwong et al., 1999; Biswas and Chan, 2010), and both Nrf1 and Nrf2 play distinct roles in the activation of ARE-driven genes (Ohtsuji et al., 2008). In this study, we demonstrated that the mRNA expression of Nrf1 was affected by neither glutamate nor PQQ, while Nrf2 was significantly decreased after glutamate injury and up-regulated with PQQ further application, which was consistent with previous studies, indicating different functions of Nrf1 and Nrf2 in response to oxidative stress.

With the change in Nrf2 expression, some ARE-containing phase II genes, including HO-1 and GCLC, were significantly increased by PQQ. These data suggested that Nrf2/ARE signaling may play important roles in the protective effects of PQQ. Both HO-1 and GCLC belong to phase II detoxifying enzymes, and their expressions are regulated through a cis-acting ARE located in their promoters. Phase II enzymes were originally recognized as xenobiotic metabolizing enzymes responsible for the elimination of xenobiotics (Jung and Kwak, 2010). HO-1 is a redox-sensitive inducible stress protein and plays a neuroprotective role against excitotoxicity damage and brain ischemia (Satoh et al., 2003), and cells from HO- $1^{-/}$mice are highly susceptible to oxidative insults (Poss and Tonegawa, 1997). GCL, formerly known as $\gamma$-glutamylcysteine synthetase, is the rate-limiting enzyme in GSH biosynthesis, and comprises a catalytic subunit (GCLC) and a modulatory (GCLM) subunit (Griffith and Mulcahy, 1999; Franklin et al., 2009). Up-regulation of HO-1 and GCL expression through the ERK/Nrf2 pathway could protect hepatocytes against oxidative stress (Yang et al., 2011). In this study, we observed that the up-regulation of these two genes was blocked by PI3K/Akt inhibition, indicating that Nrf2/ARE was, at least partly, dependent on the activation of PI3K signaling.

The cellular antioxidant system is composed of oxidant scavengers (e.g., GSH) and antioxidant enzymes (e.g., SOD). Thiol-containing compounds such as GSH and thioredoxin are oxidized by free radicals and rapidly regenerated (Yang et al., 2011). GSH is the most abundant endogenously synthesized, thiol-containing, small-molecule tripeptide, which can directly scavenge ROS in mammalian cells (Meister and Anderson, 1983). Our previous study has demonstrated that SOD activity can be up-regulated by PQQ treatment. In this study, we observed that PQQ treatment increased the GSH level and decreased ROS production in glutamate-injured hippocampal neurons. It has been reported that decreased Nrf2 transcriptional activity may cause age-related loss of GSH synthesis through modulation by GCLC, the ratelimiting enzyme in GSH biosynthesis (Suh et al., 2004). Consistent with these findings, our results showed that PQQ treatment increased the mRNA expression of GCLC in glutamate-injured hippocampal neurons, which may be responsible for the increased GSH level. However, the effects of PQQ on ROS production and GSH level did not seem to be dependent on PI3K/ Akt signaling, suggesting that some other molecules may participate in the related regulation.

In summary, we demonstrated that PQQ could protect primary cultured hippocampal neurons against glutamate-induced cell damage through the activation of Nrf2 and subsequent up-regulation of antioxidant enzyme genes including HO-1 and GCLC, which were dependent on the PI3K/Akt/GSK3 $\beta$ pathway. Moreover, the ability of PQQ to scavenge ROS, as indicated by the decreased ROS production and increased GSH level, was not totally regulated by $\mathrm{PI} 3 \mathrm{~K} / \mathrm{Akt}$ signaling, and thus, some other mechanisms may be involved. 


\section{Declaration of interest}

The authors report no conflict of interest. The authors alone are responsible for the content and writing of this paper.

\section{ACKNOWLEDGMENTS}

Research supported by the Priority Academic Program Development of Jiangsu Higher Education Institutions, the National Natural Science Foundation of China (Grant \#81171180), and the Nantong Science and Technology Bureau, Jiangsu, China (Grant \#S2010022). We thank Professor Jie Liu for assistance in manuscript preparation.

\section{REFERENCES}

Aizenman E, Hartnett KA, Zhong C, Gallop PM, et al. (1992). Interaction of the putative essential nutrient pyrroloquinoline quinone with the N-methyl-D-aspartate receptor redox modulatory site. J. Neurosci. 12: 2362-2369.

Bauerly KA, Storms DH, Harris CB, Hajizadeh S, et al. (2006). Pyrroloquinoline quinone nutritional status alters lysine metabolism and modulates mitochondrial DNA content in the mouse and rat. Biochim. Biophys. Acta 1760: 17411748.

Biswas M and Chan JY (2010). Role of Nrf1 in antioxidant response element-mediated gene expression and beyond. Toxicol. Appl. Pharmacol. 244: 16-20.

Blank V (2008). Small Maf proteins in mammalian gene control: mere dimerization partners or dynamic transcriptional regulators? J. Mol. Biol. 376: 913-925.

Chowanadisai W, Bauerly KA, Tchaparian E, Wong A, et al. (2010). Pyrroloquinoline quinone stimulates mitochondrial biogenesis through cAMP response element-binding protein phosphorylation and increased PGC-1alpha expression. J. Biol. Chem. 285: 142-152.

Cross DA, Alessi DR, Cohen P, Andjelkovich M, et al. (1995). Inhibition of glycogen synthase kinase-3 by insulin mediated by protein kinase B. Nature 378: 785-789.

Dhakshinamoorthy S, Long DJ and Jaiswal AK (2000). Antioxidant regulation of genes encoding enzymes that detoxify xenobiotics and carcinogens. Curr. Top. Cell Regul. 36: 201-216.

Franklin CC, Backos DS, Mohar I, White CC, et al. (2009). Structure, function, and post-translational regulation of the catalytic and modifier subunits of glutamate cysteine ligase. Mol. Aspects Med. 30: 86-98.

Griffith OW and Mulcahy RT (1999). The enzymes of glutathione synthesis: gamma-glutamylcysteine synthetase. $A d v$. Enzymol. Relat. Areas Mol. Biol. 73: 209-67, xii.

Hara H, Hiramatsu $\mathrm{H}$ and Adachi T (2007). Pyrroloquinoline quinone is a potent neuroprotective nutrient against 6-hydroxydopamine-induced neurotoxicity. Neurochem. Res. 32: 489-495.

Hirakawa A, Shimizu K, Fukumitsu H and Furukawa S (2009). Pyrroloquinoline quinone attenuates iNOS gene expression in the injured spinal cord. Biochem. Biophys. Res. Commun. 378: 308-312.

Huang HC, Nguyen T and Pickett CB (2002). Phosphorylation of Nrf2 at Ser-40 by protein kinase C regulates antioxidant response element-mediated transcription. J. Biol. Chem. 277: 42769-42774.

Ishii T, Itoh K, Takahashi S, Sato H, et al. (2000). Transcription factor Nrf2 coordinately regulates a group of oxidative stress-inducible genes in macrophages. J. Biol. Chem. 275: 16023-16029.

Itoh K, Chiba T, Takahashi S, Ishii T, et al. (1997). An Nrf2/small Maf heterodimer mediates the induction of phase II detoxifying enzyme genes through antioxidant response elements. Biochem. Biophys. Res. Commun. 236: 313-322.

Jung KA and Kwak MK (2010). The Nrf2 system as a potential target for the development of indirect antioxidants. Molecules 15: 7266-7291.

Kaspar JW, Niture SK and Jaiswal AK (2009). Nrf2:INrf2 (Keap1) signaling in oxidative stress. Free Radic. Biol. Med. 47: 1304-1309.

Kumazawa T, Hiwasa T, Takiguchi M, Suzuki O, et al. (2007). Activation of Ras signaling pathways by pyrroloquinoline quinone in NIH3T3 mouse fibroblasts. Int. J. Mol. Med. 19: 765-770.

Kwong M, Kan YW and Chan JY (1999). The CNC basic leucine zipper factor, Nrf1, is essential for cell survival in response to oxidative stress-inducing agents. Role for Nrf1 in gamma-ges(1) and gss expression in mouse fibroblasts. 
J. Biol. Chem. 274: 37491-37498.

Lee JM and Johnson JA (2004). An important role of Nrf2-ARE pathway in the cellular defense mechanism. J. Biochem. Mol. Biol. 37: 139-143.

Liu S, Li H, Ou YJ, Peng H, et al. (2005). Enhanced rat sciatic nerve regeneration through silicon tubes filled with pyrroloquinoline quinone. Microsurgery 25: 329-337.

Mebratu Y and Tesfaigzi Y (2009). How ERK1/2 activation controls cell proliferation and cell death: Is subcellular localization the answer? Cell Cycle 8: 1168-1175.

Meister A and Anderson ME (1983). Glutathione. Anпu. Rev. Biochem. 52: 711-760.

Misra HS, Khairnar NP, Barik A, Indira PK, et al. (2004). Pyrroloquinoline-quinone: a reactive oxygen species scavenger in bacteria. FEBS Lett. 578: 26-30.

Motohashi H, O'Connor T, Katsuoka F, Engel JD, et al. (2002). Integration and diversity of the regulatory network composed of Maf and CNC families of transcription factors. Gene 294: 1-12.

Murase K, Hattori A, Kohno M and Hayashi K (1993). Stimulation of nerve growth factor synthesis/secretion in mouse astroglial cells by coenzymes. Biochem. Mol. Biol. Int. 30: 615-621.

Niture SK, Kaspar JW, Shen J and Jaiswal AK (2010). Nrf2 signaling and cell survival. Toxicol. Appl. Pharmacol. 244: $37-42$.

Ohtsuji M, Katsuoka F, Kobayashi A, Aburatani H, et al. (2008). Nrf1 and Nrf2 play distinct roles in activation of antioxidant response element-dependent genes. J. Biol. Chem. 283: 33554-33562.

Pap M and Cooper GM (1998). Role of glycogen synthase kinase-3 in the phosphatidylinositol 3-Kinase/Akt cell survival pathway. J. Biol. Chem. 273: 19929-19932.

Poss KD and Tonegawa S (1997). Reduced stress defense in heme oxygenase 1-deficient cells. Proc. Natl. Acad. Sci. U. S. A. 94: 10925-10930.

Rojo AI, Rada P, Egea J, Rosa AO, et al. (2008). Functional interference between glycogen synthase kinase-3 beta and the transcription factor Nrf2 in protection against kainate-induced hippocampal cell death. Mol. Cell Neurosci. 39: $125-132$.

Rucker R, Chowanadisai W and Nakano M (2009). Potential physiological importance of pyrroloquinoline quinone. Altern. Med. Rev. 14: 268-277.

Salazar M, Rojo AI, Velasco D, de Sagarra RM, et al. (2006). Glycogen synthase kinase-3beta inhibits the xenobiotic and antioxidant cell response by direct phosphorylation and nuclear exclusion of the transcription factor Nrf2. J. Biol. Chem. 281: 14841-14851.

Satoh T, Baba M, Nakatsuka D, Ishikawa Y, et al. (2003). Role of heme oxygenase-1 protein in the neuroprotective effects of cyclopentenone prostaglandin derivatives under oxidative stress. Eur. J. Neurosci. 17: 2249-2255.

Scanlon JM, Aizenman E and Reynolds IJ (1997). Effects of pyrroloquinoline quinone on glutamate-induced production of reactive oxygen species in neurons. Eur. J. Pharmacol. 326: 67-74.

Shih AY, Li P and Murphy TH (2005). A small-molecule-inducible Nrf2-mediated antioxidant response provides effective prophylaxis against cerebral ischemia in vivo. J. Neurosci. 25: 10321-10335.

Stites T, Storms D, Bauerly K, Mah J, et al. (2006). Pyrroloquinoline quinone modulates mitochondrial quantity and function in mice. J. Nutr. 136: 390-396.

Suh JH, Shenvi SV, Dixon BM, Liu H, et al. (2004). Decline in transcriptional activity of Nrf2 causes age-related loss of glutathione synthesis, which is reversible with lipoic acid. Proc. Natl. Acad. Sci. U. S. A. 101: 3381-3386.

Yang YC, Lii CK, Lin AH, Yeh YW, et al. (2011). Induction of glutathione synthesis and heme oxygenase 1 by the flavonoids butein and phloretin is mediated through the ERK/Nrf2 pathway and protects against oxidative stress. Free Radic. Biol. Med. 51: 2073-2081.

Zhang Q, Shen M, Ding M, Shen D, et al. (2011). The neuroprotective action of pyrroloquinoline quinone against glutamate-induced apoptosis in hippocampal neurons is mediated through the activation of PI3K/Akt pathway. Toxicol. Appl. Pharmacol. 252: 62-72.

Zhang Y, Feustel PJ and Kimelberg HK (2006). Neuroprotection by pyrroloquinoline quinone (PQQ) in reversible middle cerebral artery occlusion in the adult rat. Brain Res. 1094: 200-206.

Zhu BQ, Simonis U, Cecchini G, Zhou HZ, et al. (2006). Comparison of pyrroloquinoline quinone and/or metoprolol on myocardial infarct size and mitochondrial damage in a rat model of ischemia/reperfusion injury. J. Cardiovasc. Pharmacol. Ther. 11: 119-128. 